\title{
DESIGN OF DUAL-BAND SLOTTED PATCH HYBRID COUPLERS BASED ON PSO ALGORITHM
}

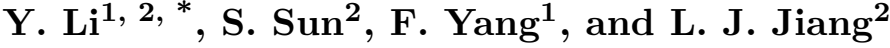 \\ ${ }^{1}$ Department of Microwave Engineering, School of Electronic Engi- \\ neering, University of Electronic Science and Technology of China, \\ Chengdu 611731, China \\ ${ }^{2}$ Department of Electrical and Electronic Engineering, The University \\ of Hong Kong, Pokfulam Road, Hong Kong, China
}

\begin{abstract}
A planar $3 \mathrm{~dB}$ patch hybrid coupler using cross and circular-shape slots is presented for a dual-band application. By inductively loading a pair of cross slots and four circular holes on a square patch, the matching and isolation performance of the miniaturized patch hybrid coupler is improved. In addition, the opencircuited shunt stubs are further installed at four ports to realize two operating bands. Since the slotted patch resonator cannot be characterized by the closed-form transmission line theory, the particle swarm optimization (PSO) algorithm is constructed and integrated with a full-wave solver to determine the variable parameters of the proposed structure. Finally, a prototype dual-band coupler operating at $915 \mathrm{MHz}$ and $1575 \mathrm{MHz}$ is designed and fabricated. Measured results show a good agreement with those obtained from simulation.
\end{abstract}

\section{INTRODUCTION}

As one of the most critical passive circuits, quadrature hybrid couplers are widely used in microwave mixers, amplifier circuits and antenna feeding networks. They provide $3 \mathrm{~dB}$ power assignments at two output ports with a $90^{\circ}$ phase difference. In the modern communication systems, various characteristics such as broadband, compact size and multiband operation are in high demand for passive circuit design. For the traditional 3-dB branch-line coupler, it has poor performance at high operating frequencies due to the parasitic effects at the Tor cross-junctions and unwanted coupling among the adjacent lowimpedance lines. To address this issue, patch hybrid coupler was

\footnotetext{
Received 28 August 2011.

* Corresponding author: Yan Li (liyaanem@gmail.com).
} 
proposed [1]. Recently, the different types of miniaturized patch hybrid couplers were proposed for the single- and dual-band applications [13]. By asymmetric loading cross slots on the patch [1] or using different etched shapes in the ground plane [2], the size of the singleband patch couplers can be effectively reduced without compromising the bandwidth. After optimizing the convex bounding around the periphery of the patch, a dual-band rectangular patch hybrid coupler was designed in [3]. Unfortunately, this kind of patch hybrid couplers cannot be characterized by the closed-form transmission line theory because of the complicated current distribution along the patch. Therefore, a number of variables have to be determined by the optimization algorithm.

In this paper, a planar 3-dB patch hybrid coupler is designed and optimized based on the concept of particle swarm optimization (PSO), which is a robust global search algorithm and derived from artificial life and evolutionary computation theory. PSO was initially proposed in 1995 [4] and has been widely used to solve general Ndimension, linear and nonlinear optimization problems. Compared with the Genetic algorithm (GA) used in $[3,5,6]$, PSO is easier to carry out and has higher efficiency [7], because just velocity and position update formulas were used in a single-object optimal model, while selection, crossover and mutation operations were needed in GA, which make it more complicated. As shown in Fig. 1, the four circular holes are symmetrically etched on the cross-slotted patch resonator [1], in order to further improve the matching and isolation performance of the patch hybrid coupler. With the help of the four open-circuited

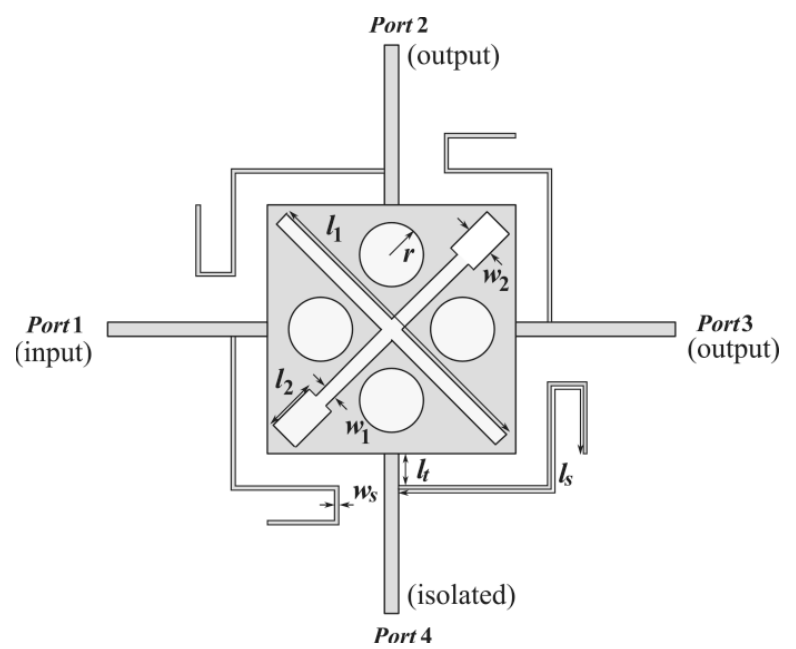

Figure 1. Configuration of the proposed dual-band coupler. 
shunt stubs $[8,9]$, a dual-band hybrid patch coupler is then carried out, aiming to achieve two operating bands at UHF RFID band $(915 \mathrm{MHz})$ and GPS band $(1575 \mathrm{MHz})$ simultaneously. By following the design guidelines of the cross-slotted patch hybrid couplers [1] and dualband branch-line couplers [9], the initial dimensions of the proposed physical topology are determined and then optimized by the PSO algorithm. The final results are verified by the experimental results of the fabricated prototype circuit.

\section{PARTICLE SWARM OPTIMIZATION}

Recently, PSO algorithm is proposed as a heuristic algorithm inspired by the choreography of a bird flock [4]. It has been widely used to solve general N-dimensional, linear and nonlinear optimization problems. PSO has the advantage of its algorithmic simplicity and robustness. PSO and Modified PSO have been used in electromagnetic community for different applications such as antenna array synthesis, antenna design and microwave passive components design [7,10-20].

The fitness function (or target function) of PSO is defined as:

$$
\min : F_{\text {fitness }}=\sum_{n}^{i=1} w_{i} \times F_{i}\left(\mathbf{x}_{k}\right)
$$

where, $n$ defines the number of objective functions, $F_{i}$ and $w_{i}$ indicate the $i$ th objective function and its weight, respectively. For each fitness function, $\mathbf{v}_{k}$ and $\mathbf{x}_{k}$ denote the velocity and position vectors in the $k$ th iteration, respectively.

The key point of this optimization algorithm is to update velocity and position iteratively as follows:

$$
\begin{aligned}
& \mathbf{v}_{k+1}=c_{0} \times \mathbf{v}_{k}+c_{1} \times \operatorname{rand} \times\left(\text { best }_{k}-\mathbf{x}_{k}\right)+c_{2} \times \operatorname{rand} \times\left(g \text { best }_{k}-\mathbf{x}_{k}\right) \\
& \mathbf{x}_{k+1}=\mathbf{x}_{k}+\mathbf{v}_{k+1}
\end{aligned}
$$

where $p$ best $_{k}$ is the best personal value, $g$ best $_{k}$ is the global best value, $c_{0}$ is the inertia weight, $c_{1}, c_{2}$ are the acceleration constants $[10,11]$.

The main steps of PSO are shown below:

Step 1. Initialize the particle positions $\left(\mathbf{x}_{k}\right)$;

Step 2. Calculate the fitness functions $(F)$ for each particle;

Step 3. Update the personal best value $\left(p\right.$ best $\left._{k}\right)$ and global best value $\left(g\right.$ best $\left._{k}\right)$;

Step 4. Update the velocity $\left(\mathbf{v}_{k}\right)$ and position $\left(\mathbf{x}_{k}\right)$ for each particle;

Step 5. Repeat step 2 to step 4 until the specified minimized fitness value is achieved. 
In order to optimize the physical dimensions of the proposed coupler in this work, the initial four-port $S$-parameters of the coupler are at first obtained from a full wave simulator $\left(\mathrm{HFSS}^{\mathrm{TM}}\right.$ ) and then transferred to the aforementioned PSO iteration as the fitness function. The flow chart of this iterative procedure is shown in Fig. 2. After obtaining the pre-specified coupler performance, the final design parameters can be determined.

\section{DUAL-BAND COUPLER DESIGN}

The physical layout of the proposed dual-band coupler is shown in Fig. 1. To minimize the size of the square patch resonator, a pair of cross slots is asymmetrically loaded on the patch. The current flow path along the patch surface, which is shown in Fig. 3, is extended and bent by the slots, and the patch resonance is thus shifted to a lower frequency for the purpose of the size reduction [1]. However, the optimized dimension in [1] is obtained manually and it is very

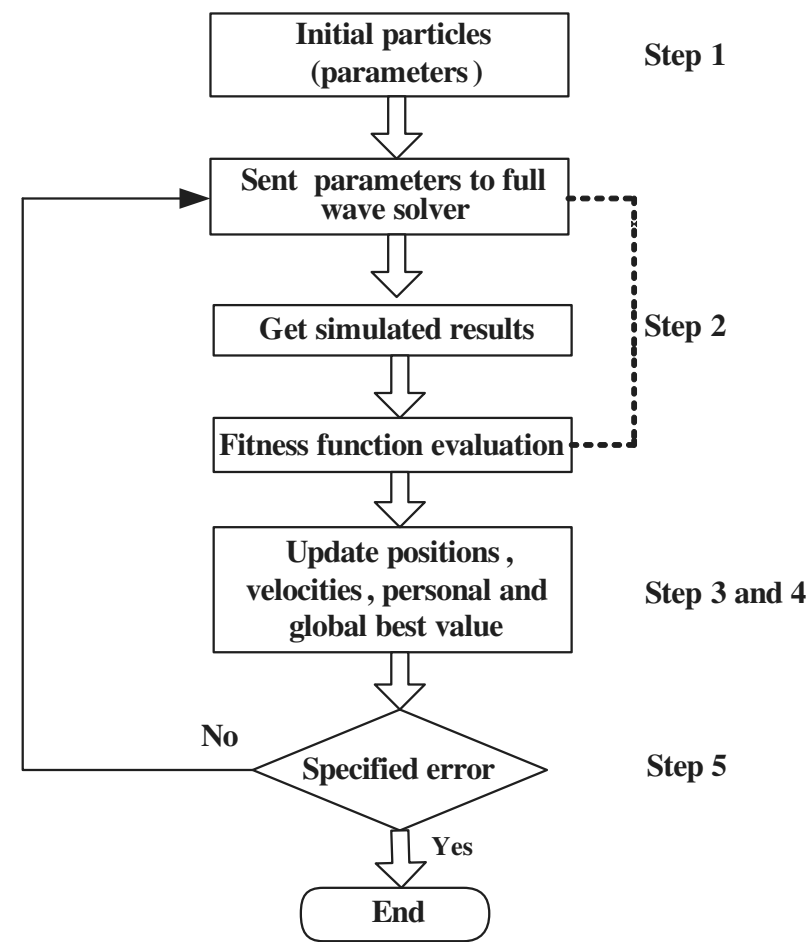

Figure 2. Flow chart of PSO integrated with full wave solver. 


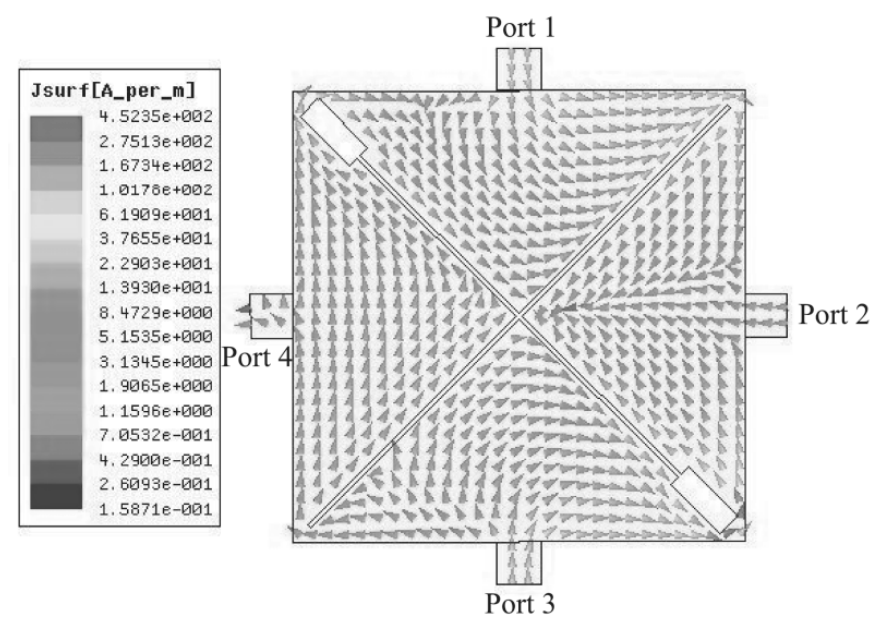

Figure 3. Current distribution for the patch coupler loading with cross slot.

time-consuming. By employing PSO, we can design the proposed coupler more efficiently. At the same time, the matching and isolation performance are also limited by the complicated current distribution along the patch. To circumvent this issue, four circular holes are further loaded on the patch symmetrically, which provide an additional degree of freedom for the performance adjustment. Since the most of the currents concentrate along the cross slots, as shown in Fig. 4, the center operating frequency of the coupler is only slightly affected by the additional circular holes. However, as mentioned in Section 1, there is no closed-form transmission line theory to characterize these loaded cross and circular holes. Hence, the PSO algorithm is employed here to reduce the rounds of the full wave simulation and find the optimized dimensions efficiently.

For the single-band coupler, the fitness function is defined as:

$$
F=w_{1} \times F_{1}+w_{2} \times F_{2}
$$

and

$$
\begin{aligned}
& F_{1}=\left|S_{21}\left(f_{d}\right)\right|-\left|S_{31}\left(f_{d}\right)\right| \\
& F_{2}=\left|S_{11}\left(f_{d}\right)\right|
\end{aligned}
$$

where $w_{1}$ and $w_{2}$ were chosen as 0.6 and 0.4 respectively in our optimization; here $f_{d}$ defines the desired frequency. According to the theory of hybrid coupler design, the ideal 3-dB hybrid has the following 


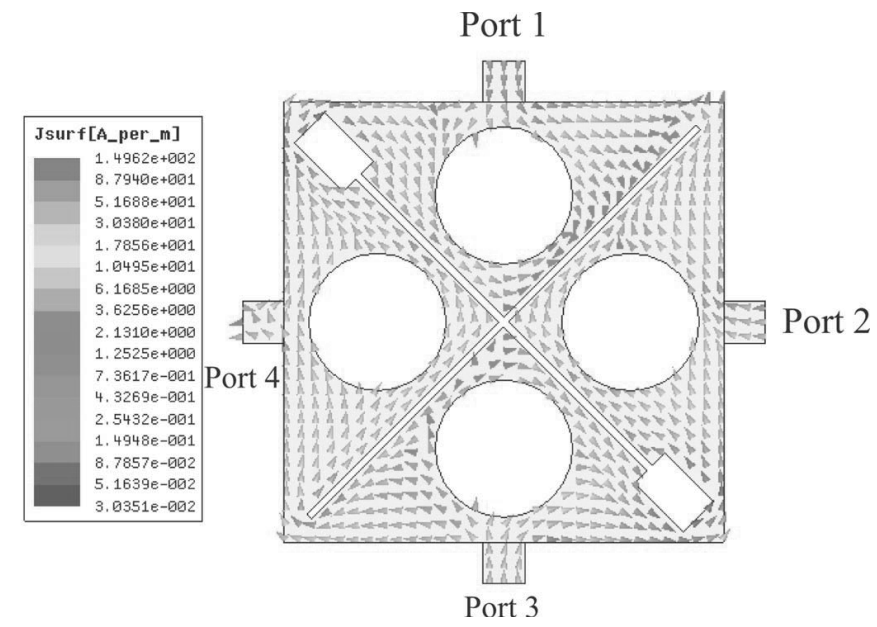

Figure 4. Current distribution for the patch coupler loading with cross slot and circular holes.

specifications:

$$
\begin{aligned}
& \left|S_{21}\right|=\left|S_{31}\right|=0.707 \\
& \left|S_{11}\right|=\left|S_{41}\right|=0 .
\end{aligned}
$$

For the single coupler design, the amplitude imbalance $\left(F_{1}\right)$ defined in $(5)$ plays a more import role than matching condition $\left(F_{2}\right)$ defined in (6). Thus, $w_{1}$ is set to 0.6 , which is slightly larger than $w_{2}$ with 0.4 . On the other hand, we just need to optimize $S_{11}$ rather than $S_{11}$ and $S_{41}$ at the same time, because $S_{41}$ will also reach the minimum when $S_{11}$ is minimized. In this design, $l_{1}=37 \mathrm{~mm}$ and $w_{1}=1 \mathrm{~mm}$ are chosen first based on the design guideline presented in [1]. By optimizing the positions and radius of the circular holes, the fitness function $F$ is targeted to be smaller than 0.1. Fig. 5(a) shows the optimized $S$ parameters of the single-band coupler with four circular holes. As expected, both matching and isolation responses are improved by loading four circular holes, as shown in Fig. 5(b).

In order to validate the compatibility of PSO algorithm for the structure with more design parameters, the dual-band coupler with four shunt open stubs are further considered here. Different from the single-band case, a number of parameters have to be optimized in the two operating frequencies at the same time. In this case, the initial value $l_{s}=33.6 \mathrm{~mm}$ is chosen about quarter-wavelength based on the design equation reported in [9]. In this design, six dimensions $\left(l_{2}, w_{2}\right.$, $r, l_{t}, w_{s}$ and $l_{s}$ ) have to be optimized at two operating frequencies 


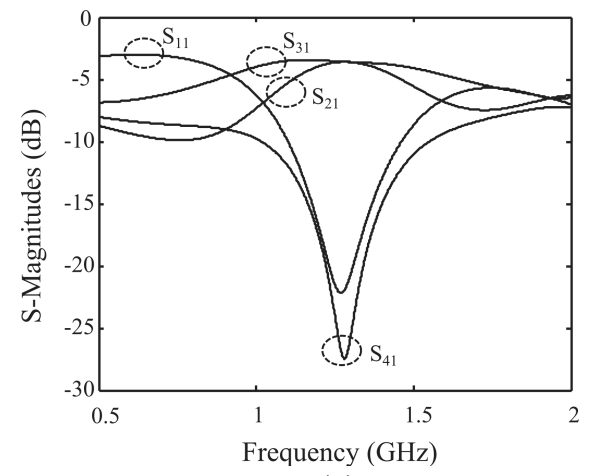

(a)

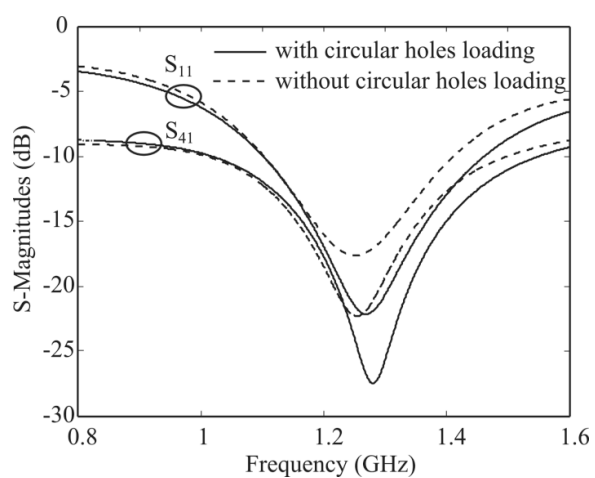

(b)

Figure 5. $S$-magnitudes of the optimized single-band coupler. (a) $S$ magnitudes. (b) Matching and isolation responses with and without circular holes.

$915 \mathrm{MHz}$ and $1.575 \mathrm{GHz}$ simultaneously. The fitness function used is similar to the previous case and is defined as:

$$
F=w_{1} \times F_{1}+w_{2} \times F_{2}
$$

and

$$
\begin{aligned}
& F_{1}=\left(\left|S_{21}\left(f_{d 1}\right)\right|-\left|S_{31}\left(f_{d 1}\right)\right|\right)+\left(\left|S_{21}\left(f_{d 2}\right)\right|-\left|S_{31}\left(f_{d 2}\right)\right|\right) \\
& F_{2}=\left|S_{11}\left(f_{d 1}\right)\right|+\left|S_{11}\left(f_{d 2}\right)\right|
\end{aligned}
$$

where $w_{1}=0.6$ and $w_{2}=0.4$; here $f_{d 1}$ defines the first desired frequency $(915 \mathrm{MHz})$ and $f_{d 2}$ defines the second desired frequency (1.575 GHz).

After optimization, the values of initial and optimized parameters are tabulated in Table 1 . The optimized amplitude response at $915 \mathrm{MHz}$ are $S_{11}=-24.78 \mathrm{~dB}, S_{21}=-3.91 \mathrm{~dB}, S_{31}=-3.56 \mathrm{~dB}$, $S_{41}=-20.13 \mathrm{~dB}$, while at $1.575 \mathrm{GHz}$, they are $S_{11}=-15.02 \mathrm{~dB}$, $S_{21}=-3.89 \mathrm{~dB}, S_{31}=-4.37 \mathrm{~dB}, S_{41}=-16.56 \mathrm{~dB}$. The phase difference at two output ports is $90.13^{\circ}$ at $915 \mathrm{MHz}$, while it is $90.24^{\circ}$ at $1.575 \mathrm{GHz}$.

Actually, the improved matching and isolation performance of the single-band coupler can effectively enhance the performance of the dual-band coupler. Fig. 6 shows the $S$-magnitudes of the dual-band coupler with and without four circular holes. In the first operating band, their responses are almost the same. However, in the second operating band with higher frequency, the matching and isolation performance can be effectively improved by installing four circular holes. 
Table 1. Initial and optimized parameters for proposed coupler.

\begin{tabular}{|c|c|c|c|}
\hline Variables in PSO & $\mathbf{x}(1)$ & $\mathbf{x}(2)$ & $\mathbf{x}(2)$ \\
\hline Parameters in couplers & $l_{2}$ & $w_{2}$ & $r$ \\
\hline Initial values & $2 \sim 8 \mathrm{~mm}$ & $2 \sim 8 \mathrm{~mm}$ & $3 \sim 6 \mathrm{~mm}$ \\
\hline Optimized value & $5 \mathrm{~mm}$ & $5.2 \mathrm{~mm}$ & $4.6 \mathrm{~mm}$ \\
\hline Variables in PSO & $\mathbf{x}(4)$ & $\mathbf{x}(5)$ & $\mathbf{x}(6)$ \\
\hline Parameters in couplers & $l_{t}$ & $l_{s}$ & $w_{s}$ \\
\hline Initial values & $1 \sim 5 \mathrm{~mm}$ & $33.6 \mathrm{~mm}$ & $0.5 \sim 1.5 \mathrm{~mm}$ \\
\hline Optimized value & $3 \mathrm{~mm}$ & $37.6 \mathrm{~mm}$ & $0.7 \mathrm{~mm}$ \\
\hline
\end{tabular}

Substrate: FR4 with thickness of $1.6 \mathrm{~mm}$, and permittivity of 4.2 .

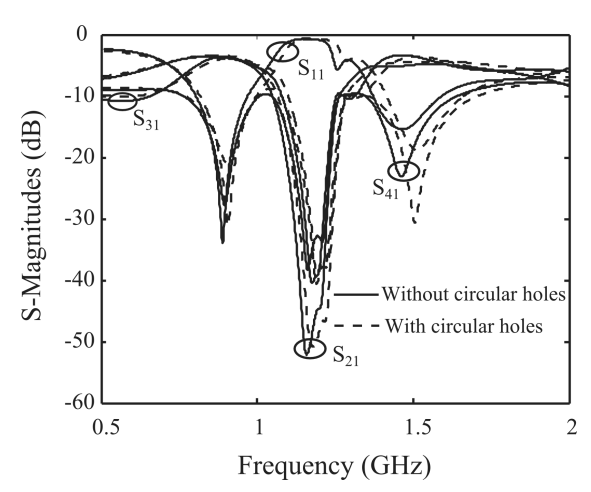

Figure 6. $S$-magnitudes of the proposed dual-band coupler with and without four circular holes.

\section{FABRICATION AND MEASURED RESULTS}

To verify the performance of our proposed dual-band coupler, an optimized prototype is finally fabricated and measured. The photograph of the fabricated coupler is shown in Fig. 7. Simulated and measured amplitude responses of the proposed coupler are shown in Fig. 8. A good agreement has been achieved between the simulated and measured results. In particular, the measured $1 \mathrm{~dB}$ amplitude imbalance achieves $17 \%$ bandwidth in the first band and $16.4 \%$ in the second band, while the measured phase differences are also within $\pm 5^{\circ}$ in these two bands, as shown in Figs. 9(a) and 9(b). 


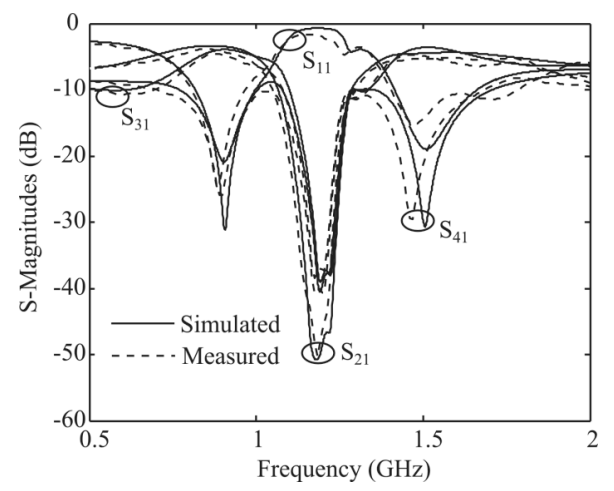

Figure 8. Simulated and measured $S$-magnitudes of the dual-band coupler.

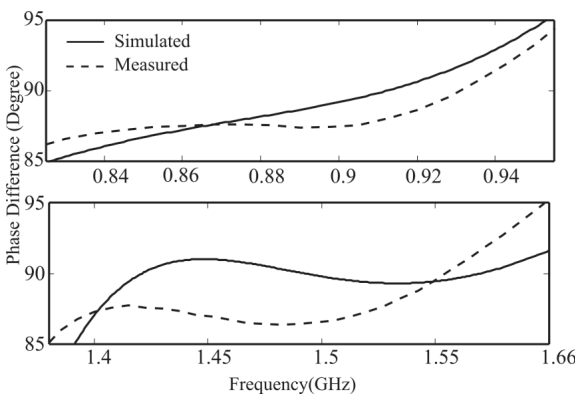

(a)

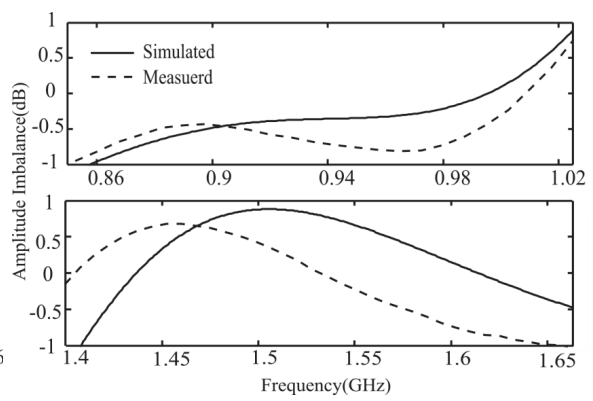

(b)

Figure 9. Simulated/measured phase difference and amplitude imbalance. (a) Phase difference. (b) Amplitude imbalance.

\section{CONCLUSION}

In this work, a dual-band patch coupler with cross and circular holes has been successfully designed and optimized at UHF RFID band and GPS band. After optimizing the fitness functions in the PSO algorithm, the variable parameters of the proposed coupler have been determined. Finally, the optimized coupler has been fabricated and verified experimentally.

\section{ACKNOWLEDGMENT}

The authors would like to thank Mr. Fangjing $\mathrm{Hu}$ (Department of Electrical and Electronic Engineering, Imperial College London, UK) 
for carrying out the full wave simulation with HFSS ${ }^{\mathrm{TM}}$. The authors also would like to thank Professor W. C. Chew for his suggestions on this paper.

\section{REFERENCES}

1. Sun, S. and L. Zhu, "Miniaturized patch hybrid couplers using asymmetrically loaded cross slots," IET Microw. Antennas Propag., Vol. 4, No. 9, 1427-1433, Sep. 2010.

2. Zheng, S. Y., S. H. Yeung, W. S. Chan, K. F. Man, and S. H. Leung, "Size reduced rectangular patch hybrid coupler using patterned ground plane," IEEE Trans. on Microw. Theory and Tech., Vol. 57, No. 1, 180-188, Jan. 2009.

3. Zheng, S. Y., S. H. Yeung, W. S. Chan, K. F. Man, S. H. Leung, and Q. Xue, "Dual-band rectangular patch hybrid coupler," IEEE Trans. on Microw. Theory and Tech., Vol. 56, No. 7, 1721-1728, Jul. 2008.

4. Kennedy, J. and R. Eberhart, "Particle swarm optimization," Proc. IEEE Int. Conf. Neural Networks, Vol. 4, 1942-1948, 2005.

5. Siakavara, K., "Novel fractal antenna arrays for satellite networks: Circular ring sierpinski carpet arrays optimized by genetic algorithms," Progress In Electromagnetics Research, Vol. 103, 115-138, 2010.

6. Reza, A. W., M. S. Sarker, and K. Dimyati, "A novel integrated mathematical approach of ray-tracing and genetic algorithm for optimizing indoor wireless coverage," Progress In Electromagnetics Research, Vol. 110, 147-162, 2010.

7. Li, Y., F. Yang, J. OuYang, Z. Nie, and H. Zhou, "Synthesis of nonuniform array antennas using particle swarm optimization," Electromagnetics, Vol. 30, No. 3, 237-245, 2010.

8. Cheng, K.-K. M. and F. L. Wong, "A novel approach to the design and implementation of dual-band compact planar $90^{\circ}$ branch-line coupler," IEEE Trans. on Microw. Theory and Tech., Vol. 52, No. 11, 2458-2463, Nov. 2004.

9. Tang, C. W. and M. G. Chen, "Design of multipassband microstrip branch-line couplers with open stubs," IEEE Trans. on Microw. Theory and Tech., Vol. 57, No. 1, 196-204, Jan. 2009.

10. Eberhart, R. C. and Y. Shi, "Particle swarm optimization: Developments, applications and resources," Proc. Congr. on Evolutionary Computation, Vol. 1, 81-86, 2001.

11. Robinson, J. and Y. Rahmat-Samii, "Particle swarm optimization 
in electromagnetic," IEEE Trans. on Antennas and Propagat, Vol. 52, No. 2, 397-407, Feb. 2004.

12. Chen, T. B., Y. L. Dong, Y. C. Jiao, and F. S. Zhang, "Synthesis of circular antenna array using crossed particle swarm optimization algorithm," Journal of Electromagnetic Waves and Applications, Vol. 20, No. 13, 1785-1795, 2006.

13. Li, W.-T., X.-W. Shi, L. Xu, and Y.-Q. Hei, "Improved GA and PSO culled hybrid algorithm for antenna array pattern sythesis," Progress In Electromagnetics Research, Vol. 80, 461-476, 2008.

14. Li, W.-T., X.-W. Shi, and W.-L. Li, "A novel spade-shaped ultrawideband antenna with dual band-notched characteristics," Journal of Electromagnetic Waves and Applications, Vol. 23, Nos. 11-12, 1455-1465, 2009.

15. Zhang, S., S.-X. Gong, and P.-F. Zhang, "A modified PSO for low sidelobe concentric ring arrays synthesis with multiple constraints," Journal of Electromagnetic Waves and Applications, Vol. 23, No. 11-12, 1535-1544, 2009.

16. Islam, M. T., M. Moniruzzaman, N. Misran, and M. N. Shakib, "Curve fitting based particle swarm optimization for UWB patch antenna," Journal of Electromagnetic Waves and Applications, Vol. 23, Nos. 17-18, 2421-2432, 2009.

17. Perez Lopez, J. R. and J. Basterrechea, "Hybrid particle swarmbased algorithms and their application to linear array synthesis," Progress In Electromagnetics Research, Vol. 90, 63-74, 2009.

18. Wang, W.-T., S.-X. Gong, Y.-J. Zhang, F.-T. Zha, J. Ling, and T. Wan, "Low RCS dipole array synthesis based on MoM-PSO hybrid algorithm," Progress In Electromagnetics Research, Vol. 94, 119$132,2009$.

19. Carro Ceballos, P. L., J. De Mingo Sanz, and P. G. Dúcar, "Radiation pattern synthesis for maximum mean effective gain with spherical wave expansions and particle swarm techniques," Progress In Electromagnetics Research, Vol. 103, 355-370, 2010.

20. Naghavi, H. A., M. Tondro-Aghmiyouni, M. Jahanbakht, and A. A. Lotfi Neyestanak, "Hybrid wideband microstrip wilkinson power divider based on lowpass filter optimized using particle swarm method," Journal of Electromagnetic Waves and Applications, Vol. 24, Nos. 14-15, 1877-1886, 2010. 